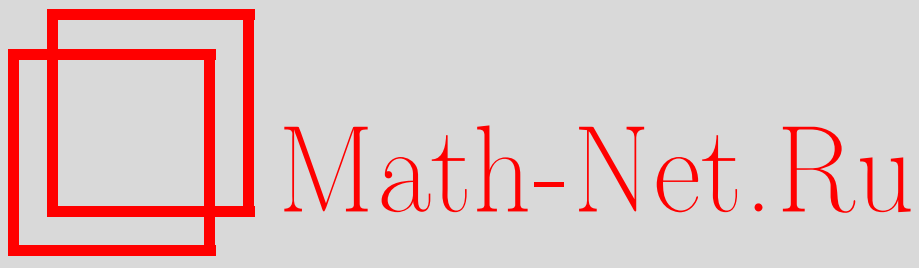

С. А. Степин, Волновые операторы для линеаризованного уравнения Больцмана в односкоростной теории переноса, Матем. сб., 2001, том 192, номер 1, 139-160

DOI: https://doi.org/10.4213/sm539

Использование Общероссийского математического портала Math-Net.Ru подразумевает, что вы прочитали и согласны с пользовательским соглашением http://www . mathnet.ru/rus/agreement

Параметры загрузки:

IP: 3.82 .47 .9

26 апреля 2023 г., $16: 25: 48$ 


\author{
С.А. Степин
}

\title{
Волновые операторы для линеаризованного уравнения Больцмана в односкоростной теории переноса
}

\begin{abstract}
В работе изучается диссипативный интегро-дифференциальный оператор $L$, возникающий при линеаризации уравнения Болњцмана в односкоростной теории переноса частиц. В предположениях, обеспечивающих конечность точечного спектра $L$, найдено скалярное кратное характеристической функции оператора $L$ и указано условие отсутствия спектральных особенностей. C помощью техники нестационарной теории рассеяния и с использованием функциональной модели Сёкефальви-Надя и раторы со свойством полноты. Изучена структура оператора $L$ в инвариантном подпространстве, отвечающем его непрерьвному спектру.

Библиография: 21 название.
\end{abstract}

Линейная теория переноса излучения является частью общей кинетической теории, которая связана с фундаментальными принципами классической статистической механики (см. [1], [2]). Используемое в этой теории основное уравнение (уравнение переноса) представляет собой вариант линеаризованного кинетического уравнения Больцмана. Математическое исследование уравнения переноса в односкоростном случае, т.е. в предположении, что изменяется лишь направление распространения излучения при постоянной энергии кванта, впервые было проведено В. С. Владимировым [3]. Обзор различных аспектов теоретических исследований уравнения Больцмана и его применений содержится в [4]. Линейной теории переноса посвящены монографии [5], [6].

В настоящей работе изучается интегро-дифференциальньй оператор, возникающий при линеаризации уравнения Больцмана в рамках односкоростной модели переноса нейтронов. Рассматривается случай плоской симметрии, когда количественные характеристики рассеяния нейтронов зависят лишь от одной пространственной координаты, скажем $x$. При этом процесс рассеяния описывается функцией распределения $\Psi(x, \mu, t)$, имеющей смысл плотности числа частиц, находящихся в момент времени $t$ в точке $c$ координатой $x$ и таких, что косинус угла между скоростью частицы и осью $x$ равен $\mu$. Линеаризованное уравнение эволюции функции распределения $\Psi(x, \mu, t)$ имеет следующий вид (см., например, [4]):

$$
\left(\frac{1}{v} \frac{\partial}{\partial t}+\mu \frac{\partial}{\partial x}+\sigma\right) \Psi(x, \mu, t)=\sigma \frac{b(x)}{2} \int_{-1}^{1} \Psi(x, \mu, t) d \mu .
$$

Здесь $v$ - скорость нейтронов, $\sigma$ - величина, обратно пропорциональная средней длине свободного пробега нейтронов между столкновениями. Не ограничивая обшности, будем считать скорость $v$ и параметр $\sigma$ единичными. Коэффициент $b(x) \in \mathrm{L}^{\infty}(\mathbb{R})$ - среднее число частищ, появляюшихся в результате столкновения нейтрона с ядром в точке с координатой $x$. 
С уравнением (1) связан оператор

$$
L=L_{0}+i V=i \mu \frac{\partial}{\partial x}+i \frac{b(x)}{2} \int_{-1}^{1} \cdot d \mu,
$$

действующий в пространстве $\mathscr{H}=\mathrm{L}^{2}(\mathbb{R} \times[-1,1])$, с областью определения $D(L)=\left\{\psi \in \mathscr{H}: \psi(\cdot, \mu)\right.$ абсолютно непрерывна для п.в. $\left.\mu \in[-1,1], L_{0} \psi \in \mathscr{H}\right\}$, где

$$
L_{0}:=i \mu \frac{\partial}{\partial x}, \quad V:=b(x) K, \quad K:=\frac{1}{2} \int_{-1}^{1} \cdot d \mu .
$$

В рассматриваемой здесь постановке вопрос об исследовании спектральных свойств и построении спектрального разложения для оператора $L$ восходит к К. О. Фридрихсу (см. [7], [8]).

В серии работ [8]-[10] были детально изучены спектральные свойства оператоpa $L$ в случае, когда функция $b(x)$ пропорциональна индикатору интервала. Для произвольной финитной неотрицательной функции $b(x) \in \mathrm{L}^{\infty}(\mathbb{R})$ в [11] показано, что непрерывный спектр оператора $L$ заполняет вешественную ось, точечный спектр состоит из нормальных полупростых собственных значений, принадлежащих $i \mathbb{R}_{+}$. В работе автора [12] показано, что при условиях $b(x) \in \mathrm{L}^{\infty}(\mathbb{R}) \cap \mathrm{L}^{2}(\mathbb{R})$ и $b(x) \geqslant 0$ для п.в. $x \in \mathbb{R}$ у оператора $L$ сохраняется указанная выше структура спектра; при условии $b(x)(\ln |x-y|)^{2} b(y) \in \mathrm{L}^{1}(\mathbb{R} \times \mathbb{R})$ суммарная кратность $N(b)$ собственных значений оператора $L$ конечна.

Для финитных функций $b(x) \in \mathrm{L}^{\infty}(\mathbb{R})$ в работе [11] в представлении функциональной модели оператора $L$ изучается компонента $L_{c}$, отвечающая его непрерывному спектру, и, в частности, получен ответ на вопрос, когда оператор $L_{c}$ подобен самосопряженному. При этом установлено, что препятствием для такого подобия служит спектральная особенность оператора $L$, а также выделено множество $\mathscr{E} \subset \mathrm{L}^{\infty}(\mathbb{R})$ такое, что оператор $L=L_{0}+i V$ (в случае финитной $\left.b(x)\right)$ имеет спектральную особенность в том и только том случае, если $b(x) \in \mathscr{E}$.

В работе [12] применительно к рассматриваемой там ситуации получено новое (по сравнению с [11]) описание “исключительного" множества $\mathscr{E}$ в терминах однопараметрического семейства операторов $L(\tau)=L_{0}+i \tau V$. А именно $b(x) \in \mathscr{E}$ в том и только том случае, если $N(\tau b)>N(b)$ для $\tau>1$. При этом требование $b(x) \notin \mathscr{E}$ снова является необходимым и достаточным условием отсутствия спектральных особенностей у оператора $L=L_{0}+i V$.

Цель настоящей работы - построение и изучение свойств волновых операторов, осушествляющих подобие $L_{c}$ и $L_{0}$ в случае нефинитной функции $b(x)$. На этом пути оказывается продуктивным соединение техники нестационарной теории рассеяния и подхода, используюшего функциональную модель Сёкефальви-Надя и Фояша. Отметим, что к оператору $L=L_{0}+i V$ метод “гладкой теории возмущений" (см. [13]) неприменим.

ТеОрема 1. Пусть $b(x) \in \mathrm{L}^{\infty}(\mathbb{R}), b(x) \geqslant 0$ при п.в. $x \in \mathbb{R}$ и сходится интеграл

$$
\int^{\infty} \frac{d t}{\sqrt{t}}\left(\int_{|x| \geqslant t} b^{2}(x) d x\right)^{1 / 2}
$$

Тогда существует волновой оператор

$$
\Omega=\mathrm{s}^{-} \lim _{t \rightarrow \infty} \exp (i t L) \exp \left(-i t L_{0}\right)
$$


Для плотного в $\mathscr{H}$ множества векторов $\varphi$ матричные әлементы $(\Omega \varphi, \psi)$, $\psi \in \mathscr{H}$, вычисляются по формуле

$$
(\Omega \varphi, \psi)=(\varphi, \psi)-\frac{1}{2 \pi} \int_{-\infty}^{\infty}\left(\sqrt{V} R_{0}(\tau+i 0) \varphi, \sqrt{V} R^{*}(\tau-i 0) \psi\right) d \tau .
$$

Здесь и далее используются следуюшие обозначения: $\sigma(A)$ - спектр оператоpa $A, \sigma_{c}(A)$ - непрерывный спектр, $\sigma_{p}(A)$ - точечный спектр оператора $A, R(\lambda):=$ $(L-\lambda I)^{-1}, R_{0}(\lambda):=\left(L_{0}-\lambda I\right)^{-1}, R^{*}(\lambda):=(R(\lambda))^{*}$.

Как показывает следуюшее предложение, в случае, когда оператор $L$ не имеет спектральных особенностей, сушествуют полные волновые операторы, реализующие подобие $L_{c}$ и $L_{0}$.

ТЕОРема 2. Пусть выполнены все условия теоремы 1 и, кроме того, $b(x)(\ln |x-y|)^{2} b(y) \in \mathrm{L}^{1}(\mathbb{R} \times \mathbb{R})$. Предположим также, что оператор $L=L_{0}+i V$ вполне несамосопряженныи и и $b(x) \notin \mathscr{E}$. Тогда $\Omega \mathscr{H}=(I-P) \mathscr{H}$, где $P$ - проектор Рисса, отвечающий $\sigma_{p}(L)$. При этом на подпространстве $(I-P) \mathscr{H}$ существует ограниченный волновой оператор

$$
\widetilde{\Omega}=\mathrm{s}-\lim _{t \rightarrow \infty} \exp \left(i t L_{0}\right) \exp (-i t L)
$$

и справедливо соотношение

$$
L_{c}=\Omega L_{0} \widetilde{\Omega},
$$

əде $L_{c}$ - сужение $L$ на подпространство $(I-P) \mathscr{H}$.

Эта теорема для рассматриваемого в работе класса функций $b(x)$ дает ответ на вопрос, поставленный Фридрихсом, в том смысле, что с помощью прямого и обратного волновых операторов $\Omega$ и $\widetilde{\Omega}$ явно строится спектральное представление оператора $L$. Требование полной несамосопряженности оператора $L$ не является ограничительным с точки зрения теории возмушений (см. [14]) и налагается для простоты изложения.

Основные результаты настоящей работы анонсированы в заметке [15].

\section{$\S 1$. Структура спектра оператора $L$}

Здесь приведена сводка результатов из работы [12], а также некоторые вспомогательные сведения, которые потребуются в дальнейшем.

Пусть $b(x) \in \mathrm{L}^{\infty}(\mathbb{R})$ и $b(x) \geqslant 0$ при п.в. $x \in \mathbb{R}$. Поскольку оператор $V \geqslant 0$ ограничен, то $L=L_{0}+i V$ - максимальный диссипативный оператор (см. [16]) и, таким образом,

$$
\sigma(L) \cap \mathbb{C}_{-}=\varnothing .
$$

Важную роль при исследовании расположения спектра $\sigma(L)$ в верхней полуплоскости $\mathbb{C}_{+}$играет следуюшее тождество:

$$
R(\lambda)=R_{0}(\lambda)-i R_{0}(\lambda) \sqrt{V}(I+Q(\lambda))^{-1} \sqrt{V} R_{0}(\lambda),
$$

где $Q(\lambda)=\sqrt{b} E(\lambda) \sqrt{b}$ и через $E(\lambda)$ обозначено сужение оператора $i K R_{0}(\lambda)$ на образ $K$, совпадающий с пространством $\mathrm{L}^{2}(\mathbb{R})$, естественным образом вложенным 
в $\mathscr{H}$. Известно (см. [8], [11]), что $Q(\lambda)$ при $\lambda \in \mathbb{C}_{+}$представляет собой интегральньй оператор с ядром

$$
q(x, y ; \lambda):=\frac{1}{2} \sqrt{b(x)} \operatorname{Ei}(i \lambda|x-y|) \sqrt{b(y)}
$$

здесь $\operatorname{Ei}(z)$ - интегральная показательная функция, определяемая формулой

$$
\operatorname{Ei}(z)=-\int_{1}^{\infty} \frac{e^{z t}}{t} d t=\ln (-z)+\gamma+\sum_{n=1}^{\infty} \frac{z^{n}}{n \cdot n !},
$$

где $\gamma$-постоянная Эйлера. Из тождества (2) следует, что

$$
\sigma(L) \cap \mathbb{C}_{+}=\left\{\lambda \in \mathbb{C}_{+}:-1 \in \sigma(Q(\lambda))\right\} .
$$

Оператор $E(\lambda), \lambda \in \mathbb{C}_{+}$, унитарно эквивалентен (см. [9]) оператору умножения на функцию

$$
\Lambda(s ; \lambda)=\frac{i}{2 s} \ln \frac{\lambda-s}{\lambda+s},
$$

причем указанная эквивалентность осуществляется преобразованием Фурье по переменной $x$. Далее, поскольку $\operatorname{Re} \lambda \cdot \operatorname{Im} \Lambda(s ; \lambda)<0$ и $(Q(\lambda) \psi, \psi)=(E(\lambda) \sqrt{b} \psi, \sqrt{b} \psi)$, то

$$
\operatorname{Re} \lambda \cdot \operatorname{Im}(Q(\lambda) \psi, \psi)<0
$$

если $\operatorname{Re} \lambda \neq 0$ и $\sqrt{b} \psi \neq 0$ как элемент $\mathrm{L}^{2}(\mathbb{R})$, и, стало быть,

$$
\sigma_{p}(L) \subset i \mathbb{R}_{+}
$$

Допустим, что $[Q(\lambda)]^{l}$ при некотором $l \in \mathbb{N}$ и всех $\lambda \in \mathbb{C}_{+}$принимает значения в классе $\mathfrak{S}_{\infty}$ компактных операторов. Тогда согласно аналитической альтернативе $\Phi$ редгольма оператор-функция $(I+Q(\lambda))^{-1}$ мероморфна в $\mathbb{C}_{+}$, причем ее вычеты в полюсах суть операторы конечного ранга. Ввиду соотношения (2) то же верно для резольвенты $R(\lambda)$, и поэтому спектр оператора $L$ в открытой верхней полуплоскости представляет собой дискретное множество конечнократных собственных значений.

Следующее утверждение дает достаточное условие компактности оператора $Q(\lambda), \lambda \in \mathbb{C}_{+}$, и, сверх того, принадлежности $Q(\lambda)$ классу $\mathfrak{S}_{2}$ операторов Гильберта-Шмидта.

ПрЕДЛОЖЕНИЕ 1 [12]. Если $b(x) \in \mathrm{L}^{\infty}(\mathbb{R}) \cap \mathrm{L}^{1}(\mathbb{R})$ u $b(x) \geqslant 0$ при п.в. $x \in \mathbb{R}$, то $Q(\lambda) \in \mathfrak{S}_{2}$ для каждого $\lambda \in \mathbb{C}_{+}$.

Доказательство этого предложения основано на следующих оценках ядра $q(x, y ; \lambda)$ интегрального оператора $Q(\lambda), \lambda \in \mathbb{C}_{+}$:

$$
\begin{array}{ll}
|q(x, y ; \lambda)| \leqslant M \sqrt{b(x) b(y)}, & |x-y| \geqslant \delta \\
|q(x, y ; \lambda)| \leqslant \frac{1}{2} \sqrt{b(x)}(|\ln | x-y||+K(\lambda)) \sqrt{b(y)}, & |x-y| \leqslant \delta
\end{array}
$$

где $M \geqslant 1, K(\lambda)=|\ln | \lambda||+$ const, $\delta=\min \left\{|\operatorname{Re} \lambda|^{-1},(\operatorname{Im} \lambda)^{-1}\right\}$, если $\operatorname{Re} \lambda \neq 0$, и $\delta=(\operatorname{Im} \lambda)^{-1}$ при $\operatorname{Re} \lambda=0$.

Отметим, что установленная в [10] применительно к рассматриваемому там случаю оценка

$$
\|Q(\lambda)\|_{\mathfrak{S}_{2}}^{2} \leqslant \text { const }|\operatorname{Re} \lambda|^{-1}
$$

для $\lambda \in \mathbb{C}_{+}$таких, что $|\operatorname{Re} \lambda| \geqslant 1$ и $\operatorname{Im} \lambda \leqslant 1$, справедлива и в предположении $b(x) \in \mathrm{L}^{\infty}(\mathbb{R}) \cap \mathrm{L}^{1}(\mathbb{R})$. Доказательство этого факта вполне аналогично проведенному в [10] в случае, когда функция $b(x)$ пропорциональна индикатору интервала. 
ПРЕДЛОЖЕНИЕ 2 [12]. Пусть $b(x) \in \mathrm{L}^{\infty}(\mathbb{R}) \cap \mathrm{L}^{2}(\mathbb{R})$ u $b(x) \geqslant 0$ nрu n.в. $x \in \mathbb{R}$. Тогда непрерывный спектр оператора $L$ заполняет вещественную ось, а точечный спектр состоит из нормальных полупростых собственных значений, принадлежсащих $i \mathbb{R}_{+}$. Суммарная кратность $N(b)$ собственных значений оператора $L$ допускает оценку

$$
N(b) \leqslant 1+\frac{1}{4} \iint_{\mathbb{R} \times \mathbb{R}} b(x)(\ln |x-y|)^{2} b(y) d x d y .
$$

\section{§2. Построение прямого волнового оператора}

Ниже с помощью метода Кука строится прямой волновой оператор $\Omega$, сплетающий $L$ и $L_{0}$, и найдено стационарное представление для матричных элементов оператора $\Omega$.

Через $U(t)$ обозначим однопараметрическое семейство операторов

$$
U(t) \psi(x, \mu)=\lim _{n \rightarrow \infty}\left(I-\frac{i L t}{n}\right)^{-n} \psi(x, \mu)
$$

(область определения $U(t), t \in \mathbb{R}$, состоит из тех $\psi \in \mathscr{H}$, для которых сушествует указанньй предел); при $t \geqslant 0$ операторы $U(t)$ образуют сжимающую полугруппу $U(t)=\exp (i t L)$. Действие унитарной группы $U_{0}(t)=\exp \left(i t L_{0}\right)$ задается следующей формулой

$$
U_{0}(t) \psi(x, \mu)=\psi(x-\mu t, \mu) .
$$

TЕOРЕMA 1. Пусть $b(x) \in \mathrm{L}^{\infty}(\mathbb{R}), b(x) \geqslant 0$ nри п.в. $x \in \mathbb{R}$ и сходится интеграл

$$
\int^{\infty} \frac{d t}{\sqrt{t}}\left(\int_{|x| \geqslant t} b^{2}(x) d x\right)^{1 / 2}
$$

Тогда существует волновой оператор $\Omega=\mathrm{s}-\lim _{t \rightarrow \infty} U(t) U_{0}(-t)$, сплетающий $L$ и $L_{0}: L \Omega=\Omega L_{0}$. Для плотного в $\mathscr{H}$ множества векторов $\varphi$ матричные әлементы $(\Omega \varphi, \psi), \psi \in \mathscr{H}$, вычисляются по формуле

$$
(\Omega \varphi, \psi)=(\varphi, \psi)-\frac{1}{2 \pi} \int_{-\infty}^{\infty}\left(\sqrt{V} R_{0}(\tau+i 0) \varphi, \sqrt{V} R^{*}(\tau-i 0) \psi\right) d \tau
$$

ДоказАТЕЛЬСтво. 1) Поскольку $\left\|U(t) U_{0}(-t)\right\| \leqslant 1, t \geqslant 0$, то достаточно доказать существование предела

$$
\lim _{t \rightarrow \infty} U(t) U_{0}(-t) \varphi
$$

для векторов $\varphi$ из плотного в $\mathscr{H}$ множества

$$
\mathscr{D}=\mathrm{C}_{0}^{\infty}(\mathbb{R} \times\{(-1,0) \cup(0,1)\}) .
$$

Согласно методу Кука (см., например, [17]) существование предела (11) обеспечивается сходимостью интеграла

$$
\int_{0}^{\infty}\left\|V U_{0}(-t) \varphi\right\| d t<\infty
$$


Здесь $\varphi \in \mathscr{D}$ и, следовательно, $\varphi(s, \mu)=0$ при $|s| \geqslant a$ и $|\mu| \leqslant \varepsilon$ для некоторых $a>0$ и $\varepsilon>0$. Положим $M=\max |\varphi(s, \mu)|$ и выполним оценку нормы вектора $V U_{0}(-t) \varphi$. При $t>0$ имеем

$$
\begin{aligned}
\left\|V U_{0}(-t) \varphi\right\|^{2} & =\frac{1}{2} \int_{\mathbb{R}} b^{2}(x)\left|\int_{-1}^{1} \varphi(x+\mu t, \mu) d \mu\right|^{2} d x \\
& \leqslant \int_{\mathbb{R}} b^{2}(x) \int_{-1}^{1}|\varphi(x+\mu t, \mu)|^{2} d \mu d x \\
& =\int_{\mathbb{R}} d s \int_{-1}^{1} b^{2}(s-\mu t)|\varphi(s, \mu)|^{2} d \mu \\
& \leqslant M^{2} \int_{-a}^{a} d s\left(\int_{-1}^{-\varepsilon}+\int_{\varepsilon}^{1}\right) b^{2}(s+\mu t) d \mu .
\end{aligned}
$$

Отсюда следует неравенство

$$
\int_{0}^{\infty}\left\|V U_{0}(-t) \varphi\right\| d t \leqslant M \sqrt{2 a} \int_{0}^{\infty} \frac{d t}{\sqrt{t}}\left\{\left(\int_{-a-t}^{a-\varepsilon t}+\int_{-a+\varepsilon t}^{a+t}\right) b^{2}(x) d x\right\}^{1 / 2}
$$

где правая часть конечна при условии сходимости интеграла (9).

В результате установлены существование и ограниченность оператора $\Omega=$ $\mathrm{s}-\lim _{t \rightarrow \infty} U(t) U_{0}(-t)$, допускающего представление

$$
\Omega=I-\int_{0}^{\infty} U(t) V U_{0}(-t) d t
$$

2) Проверим, что $\Omega$ сплетает операторы $L$ и $L_{0}$. В самом деле, для произвольного $t>0$ имеем $U(t) \Omega=\Omega U_{0}(t)$. При $t \downarrow 0$ в равенстве

$$
\frac{U(t)-I}{t} \Omega \varphi=\Omega \frac{U_{0}(t)-I}{t} \varphi, \quad \varphi \in D\left(L_{0}\right)
$$

предел справа сушествует и равен $i \Omega L_{0} \varphi$. Стало быть, существует предел слева

$$
\lim _{t \downarrow 0} \frac{U(t)-I}{t} \Omega \varphi=i L \Omega \varphi
$$

где $\Omega \varphi \in D(L)=D\left(L_{0}\right)$ и $L \Omega \varphi=\Omega L_{0} \varphi$ для $\varphi \in D\left(L_{0}\right)$.

3) Повторяя выкладки, проведенные в ходе доказательства первой части теоремы, для элементов $\varphi$ из плотного в $\mathscr{H}$ множества $\mathscr{D}$ при $t>0$ будем иметь:

$$
\left\|\sqrt{V} U_{0}( \pm t) \varphi\right\|^{2} \leqslant \frac{\mathrm{const}}{t}\left(\int_{-a-t}^{a-\varepsilon t}+\int_{-a+\varepsilon t}^{a+t}\right) b(x) d x
$$

С использованием неравенства Буняковского-Коши отсюда получаем оценку

$$
\left\|\sqrt{V} U_{0}( \pm t) \varphi\right\|^{2} \leqslant \text { const } \frac{\sqrt{t+2 a}}{t}\left\{\left(\int_{-a-t}^{a-\varepsilon t}+\int_{-a+\varepsilon t}^{a+t}\right) b^{2}(x) d x\right\}^{1 / 2}
$$


и, таким образом, $\left\|\sqrt{V} U_{0}(t) \varphi\right\| \in \mathrm{L}^{2}(\mathbb{R})$ при условии сходимости интеграла $(9)$. Учитывая известные (см. [13]) равенства

$$
\int_{\mathbb{R}}\left\|\sqrt{V} R_{0}(k \pm i \varepsilon) \varphi\right\|^{2} d k=2 \pi \int_{0}^{\infty} e^{-2 \varepsilon t}\left\|\sqrt{V} U_{0}(\mp t) \varphi\right\|^{2} d t
$$

где $\varepsilon>0$, заключаем, что

$$
\sup _{\varepsilon>0} \int_{\mathbb{R}}\left\|\sqrt{V} R_{0}(k \pm i \varepsilon) \varphi\right\|^{2} d k<\infty
$$

Как следствие этого сушествуют предельные значения $\sqrt{V} R_{0}(\kappa+i 0) \varphi$ для п.в. $\kappa \in \mathbb{R}$ и в среднем квадратичном имеет место сходимость $\sqrt{V} R_{0}(\kappa+i \varepsilon) \varphi \rightarrow$ $\sqrt{V} R_{0}(\kappa+i 0) \varphi$, когда $\varepsilon \downarrow 0$.

Далее, поскольку $L$ - максимальный диссипативньй оператор с ограниченной мнимой частью, то согласно [14] для произвольного вектора $\psi \in \mathscr{H}$ :

$$
\sup _{\varepsilon>0} \int_{\mathbb{R}}\left\|\sqrt{V} R^{*}(k-i \varepsilon) \psi\right\|^{2} d k<\infty
$$

при п.в. $\kappa \in \mathbb{R}$ определены граничные значения $\sqrt{V} R^{*}(\kappa-i 0) \psi$ и имеет место сходимость $\sqrt{V} R^{*}(\kappa-i \varepsilon) \psi \rightarrow \sqrt{V} R^{*}(\kappa-i 0) \psi$ при $\varepsilon \downarrow 0$ в среднем квадратичном.

4) Согласно (12) для произвольных векторов $\varphi, \psi \in \mathscr{H}$ выполнено равенство

$$
(\Omega \varphi, \psi)=(\varphi, \psi)-\int_{0}^{\infty}\left(\sqrt{V} U_{0}(-t) \varphi, \sqrt{V} U^{*}(t) \psi\right) d t
$$

где $U^{*}(t)=\exp \left(-i t L^{*}\right)$. В силу известной формулы, связьвающей экспоненту и резольвенту оператора, имеем

$$
\begin{gathered}
i \int_{0}^{\infty} e^{i \kappa t} e^{-\varepsilon t} \sqrt{V} U_{0}(-t) \varphi d t=\sqrt{V} R_{0}(\kappa+i \varepsilon) \varphi \\
i \int_{0}^{\infty} e^{i \kappa t} e^{-\varepsilon t} \sqrt{V} U^{*}(t) \psi d t=\sqrt{V} R^{*}(\kappa-i \varepsilon) \psi .
\end{gathered}
$$

Таким образом, $\sqrt{V} R_{0}(\kappa+i \varepsilon) \varphi$ и $\sqrt{V} R^{*}(\kappa-i \varepsilon) \psi$ суть обратные преобразования Фурье вектор-функций $i \sqrt{2 \pi} \rho(t) e^{-\varepsilon t} \sqrt{V} U_{0}(-t) \varphi$ и $i \sqrt{2 \pi} \rho(t) e^{-\varepsilon t} \sqrt{V} U^{*}(t) \psi$, где $\rho(t)$ - функция Хевисайда.

Доказательство завершается следуюшей выкладкой, в которой используется равенство Парсеваля:

$$
\begin{aligned}
((I-\Omega) \varphi, \psi) & =\lim _{\varepsilon \downarrow 0} \int_{\mathbb{R}} e^{-2 \varepsilon t}\left(\sqrt{V} U_{0}(-t) \varphi, \sqrt{V} U^{*}(t) \psi\right) \rho(t) d t \\
& =\frac{1}{2 \pi} \lim _{\varepsilon \downarrow 0} \int_{\mathbb{R}}\left(\sqrt{V} R_{0}(\kappa+i \varepsilon) \varphi, \sqrt{V} R^{*}(\kappa-i \varepsilon) \psi\right) d \kappa \\
& =\frac{1}{2 \pi} \int_{\mathbb{R}}\left(\sqrt{V} R_{0}(\kappa+i 0) \varphi, \sqrt{V} R^{*}(\kappa-i 0) \psi\right) d \kappa
\end{aligned}
$$

ЗАмечАниЕ. Для сходимости интеграла (9) достаточным является, например, следуюшее условие: $b(x)=O\left(|x|^{-\alpha}\right)$ при $|x| \rightarrow \infty$, где $\alpha>1$.

При условии малости возмушения в рамках так называемой “гладкой теории" стационарное представление типа (10) для билинейной формы волнового оператора установлено в [13]; отметим, что рассматриваемая здесь операторная модель не укладывается в схему работы [13]. 


\section{§3. Функциональная модель оператора $L$}

Для определенности далее ограничимся рассмотрением случая, когда оператор $L=L_{0}+i V$ вполне несамосопряженный, т.е. $L$ не имеет нетривиальных приводящих подпространств, на которых он индуцирует самосопряженный оператор. Дадим краткое описание функциональной модели вполне несамосопряженного диссипативного оператора применительно к $L=L_{0}+i V$. При этом будет использоваться так называемая симметричная форма записи модели (см. [14], [18]).

Обозначим $E=\operatorname{clos} \mathscr{H}(V \mathscr{H})$ и ведем в рассмотрение характеристическую функцию $S(\lambda): E \rightarrow E$ оператора $L$, задаваемую формулой

$$
S(\lambda)=I+2 i \sqrt{V}\left(L^{*}-\lambda I\right)^{-1} \sqrt{V}, \quad \lambda \in \mathbb{C}_{+} .
$$

Аналитическая в $\mathbb{C}_{+}$оператор-функция $S(\lambda)$ является там сжимаюшей и для п.в. $k \in \mathbb{R}$ имеет граничные значения $S(k):=S(k+i 0)$ в смысле сильной сходимости. При $\lambda \in \mathbb{C}_{+} \backslash \sigma(L)$ справедливо равенство

$$
S^{-1}(\lambda)=I-2 i \sqrt{V}(L-\lambda I)^{-1} \sqrt{V} .
$$

С операторньп семейством $Q(\lambda): E \rightarrow E$ характеристическая функция $S(\lambda)$ связана соотношением

$$
S(\lambda)=(I+Q(\lambda))(I-Q(\lambda))^{-1}
$$

Обозначим через $\mathfrak{L}$ гильбертово факторпространство функций на $\mathbb{R}$ со значениями в $E \oplus E$ и скалярным произведением

$$
\left\langle\left(\begin{array}{c}
f \\
g
\end{array}\right),\left(\begin{array}{c}
\widetilde{f} \\
\widetilde{g}
\end{array}\right)\right\rangle=\int_{\mathbb{R}}\left(\left(\begin{array}{cc}
I & S^{*}(k) \\
S(k) & I
\end{array}\right)\left(\begin{array}{c}
f(k) \\
g(k)
\end{array}\right),\left(\begin{array}{c}
\widetilde{f}(k) \\
\widetilde{g}(k)
\end{array}\right)\right)_{E \oplus E} d k
$$

(подразумевается, что в множестве двухкомпонентных вектор-функций проведено отождествление элементов с нулевой нормой разности). В $\mathfrak{L}$ рассмотрим подпространство

$$
\mathfrak{H}=\left\{\left(\begin{array}{l}
f \\
g
\end{array}\right) \in \mathfrak{L}: f+S^{*} g \in \mathrm{H}^{2}\left(\mathbb{C}_{-} ; E\right), S f+g \in \mathrm{H}^{2}\left(\mathbb{C}_{+} ; E\right)\right\}
$$

Здесь $\mathrm{H}^{2}\left(\mathbb{C}_{ \pm} ; E\right)$ - пространства Харди аналитических в $\mathbb{C}_{ \pm}$вектор-функций $h$ со значениями в гильбертовом пространстве $E$, для которых

$$
\sup _{\varepsilon>0} \int_{\mathbb{R}}\|h(k \pm i \varepsilon)\|^{2} d k<\infty
$$

такие функции отождествляются с классами эквивалентности своих граничных значений на вещественной оси, образующими подпространства в $\mathrm{L}^{2}(\mathbb{R} ; E)$. Непосредственно проверяется, что ортопроектор $\mathscr{P}$ на $\mathfrak{H}$ в $\mathfrak{L}$ имеет вид

$$
\mathscr{P}\left(\begin{array}{c}
f \\
g
\end{array}\right)=\left(\begin{array}{c}
f-P_{+}\left(f+S^{*} g\right) \\
g-P_{-}(S f+g)
\end{array}\right)
$$

где $P_{ \pm}$- ортопроекторы на подпространства $\mathrm{H}^{2}\left(\mathbb{C}_{ \pm} ; E\right)$ в $\mathrm{L}^{2}(\mathbb{R} ; E)$. 
Вполне несамосопряженньй оператор $L$ унитарно эквивалентен генератору сжимающей полугруппы

$$
Z_{t}=\mathscr{P} U_{t} \mid \mathfrak{H}, \quad t \geqslant 0,
$$

где $\mathscr{U}_{t}-$ унитарная группа в $\mathfrak{L}$, действующая по формуле

$$
\mathscr{U}_{t}\left(\begin{array}{l}
f \\
g
\end{array}\right)(k)=\exp (i k t)\left(\begin{array}{c}
f(k) \\
g(k)
\end{array}\right) \text {. }
$$

Генератор полугруппы $Z_{t}$ называется функииональной моделью для $L$; изометрический оператор, осуществляющий переход в модельное представление, обозначим через $J: \mathscr{H} \rightarrow \mathfrak{H}$.

Абсолютно непрерывное (внешнее) подпространство $\mathscr{N}_{e}$ диссипативного оператора $L$ определяется как замыкание в $\mathscr{H}$ множества

$$
\left\{\psi \in \mathscr{H}: \sqrt{V} R(\lambda) \psi \in \mathrm{H}^{2}\left(\mathbb{C}_{+} ; E\right)\right\} .
$$

Сингулярное (внутреннее) подпространство $\mathscr{N}_{i}$ состоит из векторов $\psi \in \mathscr{H}$ таких, что

$$
((R(k+i \varepsilon)-R(k-i \varepsilon)) \psi, \varphi) \rightarrow 0, \quad \varepsilon \downarrow 0,
$$

для всех $\varphi \in \mathscr{H}$ при п.в. $k \in \mathbb{R}$. В модельном представлении оператора $L$ имеем

$$
\begin{gathered}
J \mathscr{N}_{e}=\operatorname{clos}_{\mathfrak{H}} \mathfrak{N}, \quad \mathfrak{N}=\left\{\mathscr{P}\left(\begin{array}{c}
f \\
-S f
\end{array}\right), \sqrt{I-S^{*} S} f \in \mathrm{L}^{2}(\mathbb{R} ; E)\right\}, \\
J \mathscr{N}_{i}=\mathfrak{H} \ominus\left\{\mathscr{P}\left(\begin{array}{c}
-S^{*} g \\
g
\end{array}\right), \sqrt{I-S S^{*}} g \in \mathrm{L}^{2}(\mathbb{R} ; E)\right\} .
\end{gathered}
$$

Чтобы записать в модельном представлении волновой оператор $\Omega$, рассмотрим линеал

$$
\mathfrak{Q}=\mathscr{P}\left\{\left(\begin{array}{l}
f \\
g
\end{array}\right) \in \mathfrak{L}: f+S^{*} g+S f+g=0\right\}
$$

и, следуя [14], на элементах $\mathscr{P}\left(\begin{array}{l}f \\ g\end{array}\right) \in \mathfrak{Q}$ зададим оператор $W\left(L, L_{0}\right)$ формулой

$$
W\left(L, L_{0}\right) \mathscr{P}\left(\begin{array}{l}
f \\
g
\end{array}\right)=\mathscr{P}\left(\begin{array}{c}
f \\
-S f
\end{array}\right) .
$$

Отметим, что для $\left(\begin{array}{l}f \\ g\end{array}\right) \in \mathfrak{L}$ справедлива оценка

$$
\begin{aligned}
\|S f+g\|^{2} & \leqslant(f, f)+\left(S^{*} g, f\right)+(S f, g)+(g, g) \\
& =\left(\left(\begin{array}{cc}
I & S^{*} \\
S & I
\end{array}\right)\left(\begin{array}{l}
f \\
g
\end{array}\right),\left(\begin{array}{l}
f \\
g
\end{array}\right)\right)_{E \oplus E}
\end{aligned}
$$

и, стало быть,

$$
\left(\begin{array}{c}
f \\
-S f
\end{array}\right)-\left(\begin{array}{l}
f \\
g
\end{array}\right)=\left(\begin{array}{c}
0 \\
-(S f+g)
\end{array}\right) \in\left(\begin{array}{c}
0 \\
\mathrm{~L}^{2}(\mathbb{R} ; E)
\end{array}\right) \subset \mathfrak{L} .
$$

Таким образом, оператор $W\left(L, L_{0}\right)$ корректно определен на $\mathfrak{Q}$, причем $W\left(L, L_{0}\right) \mathfrak{Q} \subset \mathfrak{N}$.

ЛЕмма 1. В предположсениях теоремы 1 оператор $W\left(L, L_{0}\right)$ продолжается по непрерьвности с $\mathfrak{Q}$ на все $\mathfrak{H}$ и это продолжсение совпадает с оператором $J \Omega J^{-1}$. 
ДокАЗАТЕльство. Согласно известным (см. [14]) формулам, задающим действие $U_{0}(t)$ и $U(t)$ в модельном представлении, для элементов $\mathscr{P}\left(\begin{array}{l}f \\ g\end{array}\right) \in \mathfrak{Q}$ имеем

$$
\begin{aligned}
& J U_{0}(t) J^{-1} \mathscr{P}\left(\begin{array}{l}
f \\
g
\end{array}\right)=\mathscr{P} \exp (i k t)\left(\begin{array}{l}
f \\
g
\end{array}\right), \\
& J U(t) J^{-1} W\left(L, L_{0}\right) \mathscr{P}\left(\begin{array}{l}
f \\
g
\end{array}\right)=\mathscr{P} \exp (i k t)\left(\begin{array}{c}
f \\
-S f
\end{array}\right) .
\end{aligned}
$$

Поскольку $\|U(t)\| \leqslant 1$ при $t \geqslant 0$, то

$$
\begin{aligned}
& \left\|J U(t) U_{0}(-t) J^{-1} \mathscr{P}\left(\begin{array}{l}
f \\
g
\end{array}\right)-W\left(L, L_{0}\right) \mathscr{P}\left(\begin{array}{l}
f \\
g
\end{array}\right)\right\|_{\mathfrak{L}} \\
& \leqslant\left\|J U_{0}(-t) J^{-1} \mathscr{P}\left(\begin{array}{c}
f \\
g
\end{array}\right)-J U(-t) J^{-1} W\left(L, L_{0}\right) \mathscr{P}\left(\begin{array}{l}
f \\
g
\end{array}\right)\right\|_{\mathfrak{L}} \\
& \quad=\left\|\mathscr{P} \exp (-i k t)\left(\begin{array}{c}
0 \\
S f+g
\end{array}\right)\right\|_{\mathfrak{L}},
\end{aligned}
$$

где $S f+g \in \mathrm{L}^{2}(\mathbb{R} ; E)$. В силу соотношений $P_{+}+P_{-}=I$ и $P_{+} S^{*} P_{-}=\left(P_{-} S P_{+}\right)^{*}=0$ для произвольного вектора $h \in \mathrm{L}^{2}(\mathbb{R} ; E)$ справедливы равенства

$$
\mathscr{P}\left(\begin{array}{l}
0 \\
h
\end{array}\right)=\left(\begin{array}{c}
-P_{+} S^{*} h \\
P_{+} h
\end{array}\right)=\left(\begin{array}{c}
-P_{+} S^{*} P_{+} h \\
P_{+} h
\end{array}\right)=\mathscr{P}\left(\begin{array}{c}
0 \\
P_{+} h
\end{array}\right) .
$$

Учитывая это, выполним оценку

$$
\left\|\mathscr{P} \exp (-i k t)\left(\begin{array}{c}
0 \\
S f+g
\end{array}\right)\right\|_{\mathfrak{L}} \leqslant\left\|P_{+} \exp (-i k t)(S f+g)\right\|_{\mathrm{L}^{2}(\mathbb{R} ; E)}
$$

и заметим, что правая часть последнего неравенства стремится к нулю, когда $t \rightarrow+\infty$.

Таким образом, на своей области определения $\mathfrak{Q}$ оператор $W\left(L, L_{0}\right)$ представи́м в виде $W\left(L, L_{0}\right)=\mathrm{s}-\lim _{t \rightarrow \infty} J U(t) U_{0}(-t) J^{-1}$. Остается учесть, что множество

$$
J^{-1} \mathfrak{Q}=\left\{\psi \in \mathscr{H}: \sqrt{V} R_{0}(\lambda) \psi \in \mathrm{H}^{2}\left(\mathbb{C}_{ \pm} ; E\right)\right\}
$$

плотно в $\mathscr{H}$ (см. доказательство теоремы 1$)$, или, что эквивалентно, $\mathfrak{Q}$ плотно в $\mathfrak{H}$. Стало быть, $W\left(L, L_{0}\right)$ продолжается по непрерывности на все $\mathfrak{H}$, и это продолжение совпадает с $J \Omega J^{-1}$. Попутно установлено, что $\Omega \mathscr{H} \subset \mathscr{N}_{e}$. Лемма доказана.

\section{§4. Скалярное кратное характеристической функции}

Дальнейшие рассмотрения используют наличие скалярного кратного у характеристической функции $S(\lambda)$, а также и у оператор-функции $I+S(\lambda)$. Cкалярнылм кратнымм ограниченной аналитической в полуплоскости $\mathbb{C}_{+}$оператор-функции $D(\lambda)$ называется ограниченная аналитическая скалярная функция $\delta(\lambda) \not \equiv 0$ такая, что

$$
\Delta(\lambda) D(\lambda)=D(\lambda) \Delta(\lambda)=\delta(\lambda) I, \quad \operatorname{Im} \lambda>0,
$$

где $\Delta(\lambda)$ - аналитическая в $\mathbb{C}_{+}$ограниченная оператор-функция.

ПреДЛОЖЕНИЕ 3. Пусть $b(x) \in \mathrm{L}^{\infty}(\mathbb{R}), \quad b(x) \geqslant 0 \quad n p u \quad$ n..$\quad x \in \mathbb{R} u$ $b(x)(\ln |x-y|)^{2} b(y) \in \mathrm{L}^{1}(\mathbb{R} \times \mathbb{R})$. Тогда $(S(\lambda)-I) \in \mathfrak{S}_{2}, \lambda \in \mathbb{C}_{+}$, и определители $\operatorname{det}\left(I-(S(\lambda)-I)^{2}\right)$ u $\operatorname{det}\left(I-(S(\lambda)-I)^{2} / 4\right)$ являются скалярными кратными для $S(\lambda)$ и $I+S(\lambda)$ соответственно. 
ДокАЗАТЕЛЬСТво. При сделанных относительно $b(x)$ предположениях оператор $Q(\lambda)$ принадлежит классу $\mathfrak{S}_{2}$ операторов Гильберта-Шмидта при любом $\lambda \in \mathbb{C}_{+}$(см. предложение 1$)$. Далее, имеем равенство

$$
\operatorname{Re} Q(\lambda)=-\operatorname{Im} \lambda \cdot \sqrt{V} R_{0}(\bar{\lambda}) R_{0}(\lambda) \sqrt{V}: E \rightarrow E,
$$

откуда видно, что $\operatorname{Re} Q(\lambda) \leqslant 0$, когда $\lambda \in \mathbb{C}_{+}$, т.е. числовой образ оператора $Q(\lambda), \lambda \in \mathbb{C}_{+}$, принадлежит полуплоскости $\{z \in \mathbb{C}: \operatorname{Re} z \leqslant 0\}$. Оценка резольвенты оператора через расстояние до его числового образа применительно к $Q(\lambda)$ приводит к неравенству $\left\|(I-Q(\lambda))^{-1}\right\| \leqslant 1$, где $\lambda \in \mathbb{C}_{+}$. Поскольку $S(\lambda)-I=2 Q(\lambda)(I-Q(\lambda))^{-1}$ в силу формулы $(13)$, из сказанного выше следует, что $(S(\lambda)-I) \in \mathfrak{S}_{2}, \lambda \in \mathbb{C}_{+}$, причем

$$
\|S(\lambda)-I\|_{\mathfrak{S}_{2}} \leqslant 2\|Q(\lambda)\|_{\mathfrak{S}_{2}}, \quad \lambda \in \mathbb{C}_{+} .
$$

Для доказательства второй части сформулированного утверждения достаточно убедиться, что

$$
\sup _{\lambda \in \mathbb{C}_{+}}\|S(\lambda)-I\|_{\mathfrak{S}_{2}}<\infty
$$

при этом согласно [19] оператор-функции $S(\lambda)$ и $I+S(\lambda)$ обладают скалярными кратными $\operatorname{det}\left(I-(S(\lambda)-I)^{2}\right)$ и $\operatorname{det}\left(I-(S(\lambda)-I)^{2} / 4\right)$ соответственно. Проверка равномерной ограниченности $\mathfrak{S}_{2}$-нормы оператора $S(\lambda)-I$ осуществляется по той же схеме, что и в случае финитной функции $b(x)$ (см. [11]).

Для значений $\lambda \in \mathbb{C}_{+},|\operatorname{Re} \lambda| \geqslant 1, \operatorname{Im} \lambda \leqslant 1$, в силу оценки (8) норма $\|Q(\lambda)\|_{\mathfrak{S}_{2}}$ равномерно ограничена. Если $\operatorname{Im} \lambda \geqslant 1$, то $|\operatorname{Ei}(i \lambda|x-y|)| \leqslant|\operatorname{Ei}(-|x-y|)|$ и, следовательно, справедливо неравенство

$$
\|Q(\lambda)\|_{\mathfrak{S}_{2}} \leqslant\|Q(i)\|_{\mathfrak{S}_{2}}, \quad \operatorname{Im} \lambda \geqslant 1 .
$$

Согласно (15) одновременно с $\|Q(\lambda)\|_{\mathfrak{S}_{2}}$ величина $\|S(\lambda)-I\|_{\mathfrak{S}_{2}}$ ограничена равномерно для значений $\lambda \in \mathbb{C}_{+}$таких, что $\operatorname{Im} \lambda \geqslant 1$ или $|\operatorname{Re} \lambda| \geqslant 1$.

Докажем теперь, что норма $\|S(\lambda)-I\|_{\mathfrak{S}_{2}}$ ограничена, когда $\lambda \rightarrow 0, \lambda \in \mathbb{C}_{+}$. Для этого рассмотрим интегральный оператор $\widetilde{Q}(\lambda)$ с ядром

$$
\widetilde{q}(x, y ; \lambda):=q(x, y ; \lambda) \chi(x-y),
$$

где $q(x, y ; \lambda)$ - ядро интегрального оператора $Q(\lambda), \chi$ - индикатор промежутка $\left[-|\lambda|^{-1 / 2},|\lambda|^{-1 / 2}\right]$. Согласно формуле (3) ядро $\widetilde{q}(x, y ; \lambda)$ допускает представление

$$
\begin{aligned}
\widetilde{q}(x, y ; \lambda)= & \frac{1}{2} \sqrt{b(x)}(\ln |x-y|) \sqrt{b(y)} \chi(x-y) \\
& +\frac{1}{2} \sqrt{b(x)}(\ln (-i \lambda)+\gamma) \sqrt{b(y)} \chi(x-y) \\
& -\frac{1}{2} \sqrt{b(x)} \operatorname{Ein}(-i \lambda|x-y|) \sqrt{b(y)} \chi(x-y) ;
\end{aligned}
$$

здесь $\operatorname{Ein}(z)$ - дополнительная интегральная показательная функция, определяемая формулой

$$
\operatorname{Ein}(z)=\int_{0}^{z} \frac{1-e^{-t}}{t} d t=-\sum_{n=1}^{\infty} \frac{(-z)^{n}}{n \cdot n !}
$$


Покажем, что $\|Q(\lambda)-\widetilde{Q}(\lambda)\|_{\mathfrak{S}_{2}} \rightarrow 0$ при $\lambda \rightarrow 0, \lambda \in \mathbb{C}_{+}$. Вследствие неравенств (6) и (7) имеем

$$
\begin{aligned}
&|q(x, y ; \lambda)-\widetilde{q}(x, y ; \lambda)|^{2} \leqslant b(x)(\ln |x-y|)^{2} b(y)\{1-\chi(x-y)\} \\
&+\widetilde{K}(\lambda)^{2} b(x) b(y)\{1-\chi(x-y)\}
\end{aligned}
$$

где $\widetilde{K}(\lambda)=O(|\ln | \lambda||), \lambda \rightarrow 0$. Поскольку $b(x)(\ln |x-y|)^{2} b(y) \in \mathrm{L}^{1}(\mathbb{R} \times \mathbb{R})$, то

$$
\iint_{\mathbb{R} \times \mathbb{R}} b(x)(\ln |x-y|)^{2} b(y)\{1-\chi(x-y)\} d x d y \rightarrow 0, \quad \lambda \rightarrow 0 .
$$

Кроме того, при $|\lambda|<1$ имеем

$$
\begin{aligned}
& \iint_{\mathbb{R} \times \mathbb{R}} b(x) b(y)\{1-\chi(x-y)\} d x d y \\
& \quad=\iint_{|x-y|>|\lambda|^{-1 / 2}} b(x) b(y) d x d y \\
& \quad \leqslant 4(\ln |\lambda|)^{-2} \iint_{|x-y|>|\lambda|^{-1 / 2}} b(x)(\ln |x-y|)^{2} b(y) d x d y
\end{aligned}
$$

и, стало быть,

$$
\widetilde{K}(\lambda)^{2} \iint_{\mathbb{R} \times \mathbb{R}} b(x) b(y)\{1-\chi(x-y)\} d x d y \rightarrow 0, \quad \lambda \rightarrow 0 .
$$

Таким образом, получаем, что

$$
\|Q(\lambda)-\widetilde{Q}(\lambda)\|_{\mathfrak{S}_{2}}^{2}=\iint_{\mathbb{R} \times \mathbb{R}}|q(x, y ; \lambda)-\widetilde{q}(x, y ; \lambda)|^{2} d x d y \rightarrow 0
$$

при $\lambda \rightarrow 0, \lambda \in \mathbb{C}_{+}$.

Далее, представим разность $S(\lambda)-I$ в виде

$$
S(\lambda)-I=2 \widetilde{Q}(\lambda)(I-Q(\lambda))^{-1}+2(Q(\lambda)-\widetilde{Q}(\lambda))(I-Q(\lambda))^{-1} .
$$

Из предыдущего следует, что второе слагаемое в правой части (17) стремится к нулю относительно $\mathfrak{S}_{2}$-нормы, когда $\lambda \rightarrow 0, \lambda \in \mathbb{C}_{+}$. Рассмотрим первое слагаемое из правой части (17). В соответствии с формулой (16) имеем

$$
2 \widetilde{Q}(\lambda)(I-Q(\lambda))^{-1}=(A(\lambda)-B(\lambda))(I-Q(\lambda))^{-1}+P(\lambda),
$$

где $\operatorname{rank} P(\lambda)=1, A(\lambda)$ и $B(\lambda)$ - интегральные операторы с ядрами

$$
\sqrt{b(x)}(\ln |x-y|) \sqrt{b(y)} \chi(x-y) \text { и } \sqrt{b(x)} \operatorname{Ein}(-i \lambda|x-y|) \sqrt{b(y)} \chi(x-y)
$$

соответственно. При этом

$$
\|A(\lambda)\|_{\mathfrak{S}_{2}}^{2} \leqslant \iint_{\mathbb{R} \times \mathbb{R}} b(x)(\ln |x-y|)^{2} b(y) d x d y<\infty
$$


и, кроме того,

$$
\begin{aligned}
\|B(\lambda)\|_{\mathfrak{S}_{2}}^{2} & \leqslant \iint_{|x-y| \leqslant|\lambda|-1 / 2} b(x)\left(e^{|\lambda||x-y|}-1\right) b(y) d x d y \\
& \leqslant\left(e^{|\lambda|^{1 / 2}}-1\right)\left(\int_{\mathbb{R}} b(x) d x\right)^{2} \rightarrow 0, \quad \lambda \rightarrow 0 .
\end{aligned}
$$

Поскольку $\left\|(I-Q(\lambda))^{-1}\right\| \leqslant 1, \lambda \in \mathbb{C}_{+}$, то вследствие (18) справедливо неравенство

$$
\|P(\lambda)\| \leqslant 2\left\|\widetilde{Q}(\lambda)(I-Q(\lambda))^{-1}\right\|+\|A(\lambda)\|_{\mathfrak{S}_{2}}+\|B(\lambda)\|_{\mathfrak{S}_{2}} .
$$

Учитывая, что норма левой части в соотношении (17) не превосходит 2 , приходим к заключению об ограниченности величины $\left\|\widetilde{Q}(\lambda)(I-Q(\lambda))^{-1}\right\|$ в окрестности $\lambda=0$. Стало быть, норма $\|P(\lambda)\|_{\mathfrak{S}_{2}}=\|P(\lambda)\|$, а вместе с ней и $\|S(\lambda)-I\|_{\mathfrak{S}_{2}}$, остается ограниченной при $\lambda \rightarrow 0$.

Остается показать, что величина $\|S(\lambda)-I\|_{\mathfrak{S}_{2}}$ равномерно ограничена, если значения $\lambda \in \mathbb{C}_{+}$отделены от 0 и $\infty$; при этом ввиду неравенства (15) достаточно проверить ограниченность нормы $\|Q(\lambda)\|_{\mathfrak{S}_{2}}$. Пусть $q_{n}(x, y ; \lambda)=q(x, y ; \lambda)$, если $|x| \leqslant n,|y| \leqslant n$, и $q_{n}(x, y ; \lambda)=0$, если $|x|>n$ или $|y|>n$. Интегральный оператор $Q_{n}(\lambda)$ с ядром $q_{n}(x, y ; \lambda)$ как функция со значениями в $\mathfrak{S}_{2}$ допускает (см. [11]) аналитическое продолжение на открытое множество, содержащее $\mathbb{R} \backslash\{0\}$, и $\left\|Q(\lambda)-Q_{n}(\lambda)\right\|_{\mathfrak{S}_{2}} \rightarrow 0$ при $n \rightarrow \infty$ равномерно по $\lambda \in \mathbb{C}_{+}$, если значения $\lambda$ отделены от 0 и $\infty$ (в силу оценок (6) и (7)). Поэтому оператор-функция $Q(\lambda) \in \mathfrak{S}_{2}$, $\lambda \in \mathbb{C}_{+}$, продолжается по непрерьвности на $\mathbb{R} \backslash\{0\}$, причем $Q(\kappa+i 0) \in \mathfrak{S}_{2}$.

Таким образом, норма $\|Q(\lambda)\|_{\mathfrak{S}_{2}}$ и одновременно $\|S(\lambda)-I\|_{\mathfrak{S}_{2}}$ ограничены равномерно, если значения $\lambda \in \mathbb{C}_{+}$отделены от 0 и $\infty$. Доказательство закончено. Попутно было установлено сушествование предельных значений $S(\kappa+i 0)$ для всех $\kappa \in \mathbb{R} \backslash\{0\}$.

\section{§5. Условие отсутствия спектральных особенностей}

Следуя [19], назовем точку $\lambda_{0}$ непрерывного спектра оператора $L$ спектральной особенностью, если

$$
\sup _{\left|\lambda-\lambda_{0}\right|<\varepsilon}\left\|S^{-1}(\lambda)\right\|=\infty
$$

для произвольного $\varepsilon>0$. Ниже указаны условия на коэффициент $b(x)$, при которых оператор $L=L_{0}+i V$ не имеет спектральных особенностей и предельные значения оператор-функции $S^{-1}(\lambda)$ на вешественной оси равномерно ограничены. При этом "исключительным" является подмножество $\mathscr{E} \subset \mathrm{L}^{\infty}(\mathbb{R}) \cap \mathrm{L}^{1}(\mathbb{R})$, выделенное следующим условием

$$
0 \leqslant b(x) \in \mathscr{E} \Longleftrightarrow \liminf _{\varepsilon \downarrow 0}\{-1, \sigma(Q(i \varepsilon))\}=0 .
$$

УТВЕРЖДЕНИЕ [12]. Если $b(x) \notin \mathscr{E}$, то величина $N(\tau b)$ постоянна в ожрестности значения $\tau=1$; если же $b(x) \in \mathscr{E}$, то $N(\tau b)>N(b)$ nри $\tau>1$.

Согласно сформулированному утверждению увеличение числа собственных значений у оператора $L(\tau)=L_{0}+i \tau V$ с ростом $\tau$ происходит при тех и только тех значениях параметра $\tau$, для которых $\tau b \in \mathscr{E}$. А именно существует счетньй набор $\left\{\tau_{n}\right\}$ такой, что $\tau b \in \mathscr{E}$ в том и только том случае, если $\tau \in\left\{\tau_{n}\right\}$, и рождение новых собственных значений оператора $L(\tau)$ происходит из нуля при $\tau=\tau_{n}$. Отметим, что сама точка $\lambda=0$ не является собственным значением оператора $L\left(\tau_{n}\right)$. 
ПРЕДЛОЖЕНИЕ 4. Пусть выполнены условия предложсения 3. Если функиия $b(x)$ не принадлежсит множеству $\mathscr{E}$, то оператор-функция $S(\kappa)$ обратима при $\kappa \neq 0$ u

$$
\sup _{\mathbb{R} \backslash\{0\}}\left\|S^{-1}(\kappa)\right\|<\infty
$$

ДокАЗАТЕЛьСтво. Ввиду соотношения (13) для $\lambda \in \mathbb{C}_{+} \backslash \sigma(L)$ имеем

$$
S^{-1}(\lambda)=2(I+Q(\lambda))^{-1}-I
$$

Ниже будет установлена ограниченность нормы $\left\|(I+Q(\lambda))^{-1}\right\|$ в полосе $\Pi=$ $\{\lambda \in \mathbb{C}: 0<\operatorname{Im} \lambda \leqslant \delta\}$, где $\delta \in(0,1)$ выбрано так, что $\Pi \cap \sigma_{p}(L)=\varnothing$. Такой выбор $\delta$ возможен, поскольку $N(b)<\infty$ при условии $b(x)(\ln |x-y|)^{2} b(y) \in \mathrm{L}^{1}(\mathbb{R} \times \mathbb{R})($ см. предложение 2). В силу оценки (8) найдется $R>0$ такое, что норма $\left\|(I+Q(\lambda))^{-1}\right\|$ равномерно ограничена, если $|\operatorname{Re} \lambda|>R$ и $0<\operatorname{Im} \lambda \leqslant \delta$.

Рассмотрим теперь оператор-функцию $(I+Q(\lambda))^{-1}$ вблизи нуля. Покажем сначала, что

$$
\operatorname{Re} Q(\lambda)-Q(i|\lambda|) \rightarrow 0
$$

и величина $\|\operatorname{Im} Q(\lambda)\|$ ограничена, когда $\lambda \rightarrow 0$. Как видно из представления (16) ядра $\widetilde{Q}(\lambda)$, для $\lambda \in \mathbb{C}_{+}$справедливы неравенства

$$
\begin{gathered}
\|\operatorname{Re} \widetilde{Q}(\lambda)-\widetilde{Q}(i|\lambda|)\| \leqslant\|B(\lambda)\|+\|B(i|\lambda|)\|, \\
\|\operatorname{Im} \widetilde{Q}(\lambda)\| \leqslant \int_{\mathbb{R}} b(x) d x+\|B(\lambda)\|
\end{gathered}
$$

где $B(\lambda)$ - оператор с ядром $\sqrt{b(x)} \operatorname{Ein}(-i \lambda|x-y|) \sqrt{b(y)} \chi(x-y)$. Поскольку $\|B(\lambda)\| \rightarrow 0$ и $\|Q(\lambda)-\widetilde{Q}(\lambda)\| \rightarrow 0$, когда $\lambda \rightarrow 0, \lambda \in \mathbb{C}_{+}$(см. доказательство предложения 3), отсюда следует, что оператор-функция $\operatorname{Im} Q(\lambda)$ равномерно ограничена в $\mathbb{C}_{+}$вблизи точки $\lambda=0$ и $\operatorname{Re} Q(\lambda)-Q(i|\lambda|) \rightarrow 0$ относительно операторной нормы при $\lambda \rightarrow 0$.

Допустим, что норма $\left\|(I+Q(\lambda))^{-1}\right\|$ неограничена, когда $\lambda \rightarrow 0$, т.е. сушествуют последовательности $\left\{\varphi_{n}\right\} \subset E,\left\|\varphi_{n}\right\|=1$, и $\left\{\lambda_{n}\right\} \subset \mathbb{C}_{+}$такие, что $\lambda_{n} \rightarrow 0$ И

$$
\left(I+Q\left(\lambda_{n}\right)\right) \varphi_{n} \rightarrow 0, \quad n \rightarrow \infty .
$$

При этом $\left(\operatorname{Im} Q\left(\lambda_{n}\right) \varphi_{n}, \varphi_{n}\right) \rightarrow 0$, когда $n \rightarrow \infty$. Принимая во внимание равномерную ограниченность $\operatorname{Im} Q(\lambda)$ вблизи нуля и свойство (5) знакоопределенности $\operatorname{Im} Q(\lambda)$ (см. $\S 1)$, заключаем, что $\operatorname{Im} Q\left(\lambda_{n}\right) \varphi_{n} \rightarrow 0, n \rightarrow \infty$. Тогда $\left(I+\operatorname{Re} Q\left(\lambda_{n}\right)\right) \varphi_{n} \rightarrow 0$ и одновременно

$$
\left(I+Q\left(i\left|\lambda_{n}\right|\right)\right) \varphi_{n} \rightarrow 0, \quad n \rightarrow \infty
$$

С учетом самосопряженности операторов $Q(i t), t \in \mathbb{R}_{+}$, будем иметь

$$
\operatorname{dist}\left\{-1, \sigma\left(Q\left(i\left|\lambda_{n}\right|\right)\right)\right\}=\left\|\left(I+Q\left(i\left|\lambda_{n}\right|\right)\right)^{-1}\right\|^{-1} \rightarrow 0, \quad n \rightarrow \infty,
$$

что противоречит условию $b(x) \notin \mathscr{E}$. Итак, при сделанных предположениях существует $\varepsilon>0$ такое, что оператор-функция $(I+Q(\lambda))^{-1}$ равномерно ограничена для $\lambda \in \mathbb{C}_{+},|\lambda|<\varepsilon$. 
Далее воспользуемся тем фактом (см. доказательство предложения 3), что оператор-функция $Q(\lambda): E \rightarrow E, \lambda \in \mathbb{C}_{+}$, продолжается по непрерьвности на $\mathbb{R} \backslash\{0\}$, причем $Q(\kappa+i 0) \in \mathfrak{S}_{\infty}, \kappa \neq 0$. Свойство $(5)$ знакоопределенности $\operatorname{Im} Q(\lambda)$, $\operatorname{Re} \lambda \neq 0$, распространяется на предельные значения $Q(\kappa+i 0)$ и, следовательно, $\operatorname{ker}(I+Q(\kappa+i 0))=\{0\}$, если $\kappa \neq 0$. Таким образом, оператор $(I+Q(\lambda))$ обратим для всех $\lambda$ из замькания П, за исключением точки $\lambda=0$, и, стало быть, норма $\left\|(I+Q(\lambda))^{-1}\right\|$ равномерно ограничена, если значения $\lambda \in$ П отделены от 0 и $\infty$.

Суммируя сказанное, с учетом (19) можно утверждать, что оператор-функция $S(\kappa)$ обратима при $\kappa \neq 0$ и справедлива оценка

$$
\sup _{\mathbb{R} \backslash\{0\}}\left\|S^{-1}(\kappa)\right\| \leqslant 1+2 \sup _{\lambda \in \Pi}\left\|(I+Q(\lambda))^{-1}\right\|<\infty .
$$

Отметим, что условие $b(x) \notin \mathscr{E}$ использовалось лишь при доказательстве ограниченности величины $\left\|S^{-1}(\kappa)\right\|$ в окрестности нуля.

\section{§6. Существование левого обратного к $\Omega$}

В представлении функциональной модели зададим оператор $W\left(L_{0}, L\right)$ следующим образом (см. [14]). Область определения $W\left(L_{0}, L\right)$ состоит из тех элементов $\mathscr{P}\left(\begin{array}{l}f \\ g\end{array}\right) \in \mathfrak{N}$, для которых найдется единственная вектор-функция $h$ такая, что $\mathscr{P}\left(\begin{array}{l}f \\ h\end{array}\right) \in \mathfrak{Q}$ и

$$
W\left(L_{0}, L\right) \mathscr{P}\left(\begin{array}{l}
f \\
g
\end{array}\right)=\mathscr{P}\left(\begin{array}{l}
f \\
h
\end{array}\right) .
$$

Лемма 2. Пусть выполнены условия предложсения 3 и $b(x) \notin \mathscr{E}$. Тогда оператор $W\left(L_{0}, L\right)$ определен и ограничен на множсестве, плотном в $\mathfrak{N}$, а замыкание $\widetilde{\Omega}$ оператора $J^{-1} W\left(L_{0}, L\right) J$ представляет собой левый обратныи $\kappa \Omega$.

ДокАЗАТЕльство. Согласно предложению 3 сушествует ограниченная аналитическая в $\mathbb{C}_{+}$оператор-функция $\Delta(\lambda): E \rightarrow E$ такая, что

$$
\Delta(\lambda)(I+S(\lambda))=(I+S(\lambda)) \Delta(\lambda)=d(\lambda) I
$$

где $d(\lambda)=\operatorname{det}\left(I-(S(\lambda)-I)^{2} / 4\right)$. Одновременно для $\lambda \in \mathbb{C}_{+}$и почти всюду на вещественной оси справедливы соотношения

$$
\Delta^{*}(\lambda)\left(I+S^{*}(\lambda)\right)=\left(I+S^{*}(\lambda)\right) \Delta^{*}(\lambda)=\overline{d(\lambda)} I,
$$

причем $d(k+i 0) \neq 0$ для п.в. $k \in \mathbb{R}$. Отсюда следует, что компонента $h$ в правой части (20) однозначно определяется условием $\mathscr{P}\left(\begin{array}{l}f \\ h\end{array}\right) \in \mathfrak{Q}$.

Покажем (ср. с [14]), что оператор $W\left(L_{0}, L\right)$ определен на векторах из подпространства $\mathfrak{N}$, имеющих вид $\mathscr{P} \theta_{n}\left(\begin{array}{l}f \\ g\end{array}\right)$, где $g=-S f$ и $\sqrt{I-S^{*} S} f \in \mathrm{L}^{2}(\mathbb{R} ; E)$, а скалярная функция $\theta_{n}(k)$ - индикатор множества

$$
\left\{k \in \mathbb{R}:|d(k+i 0)|>\frac{1}{n}\right\}, \quad n \in \mathbb{N} .
$$


Обозначим $Y_{n}(k):=\theta_{n}(k) \Delta^{*}(k+i 0) / \overline{d(k+i 0)}$ и заметим, что

$$
\left(\begin{array}{c}
\theta_{n} f \\
-Y_{n}(I+S) f
\end{array}\right) \in \mathfrak{L}
$$

всякий раз, когда $\sqrt{I-S^{*} S} f \in \mathrm{L}^{2}(\mathbb{R} ; E)$. В самом деле, $\theta_{n}\left(\begin{array}{c}f \\ -S f\end{array}\right) \in \mathfrak{L}$ и в силу (21) имеем

$$
\left(\begin{array}{c}
\theta_{n} f \\
-Y_{n}(I+S) f
\end{array}\right)-\theta_{n}\left(\begin{array}{c}
f \\
-S f
\end{array}\right)=\left(\begin{array}{c}
0 \\
-Y_{n}\left(I-S^{*} S\right) f
\end{array}\right) \in\left(\begin{array}{c}
0 \\
\mathrm{~L}^{2}(\mathbb{R} ; E)
\end{array}\right) \subset \mathfrak{L} .
$$

Кроме того, согласно (21) вьполнено соотношение

$$
(I+S) \theta_{n} f-\left(I+S^{*}\right) Y_{n}(I+S) f=0
$$

и, таким образом,

$$
W\left(L_{0}, L\right): \mathscr{P} \theta_{n}\left(\begin{array}{c}
f \\
-S f
\end{array}\right) \mapsto \mathscr{P}\left(\begin{array}{c}
\theta_{n} f \\
-Y_{n}(I+S) f
\end{array}\right) \in \mathfrak{Q} .
$$

Поскольку $d(k+i 0) \neq 0$ при п.в. $k \in \mathbb{R}$, множество векторов вида $\mathscr{P} \theta_{n}\left(\begin{array}{c}f \\ -S f\end{array}\right)$, $\sqrt{I-S^{*} S} f \in \mathrm{L}^{2}(\mathbb{R} ; E), n \in \mathbb{N}$, на которых определен оператор $W\left(L_{0}, L\right)$, плотно в $\mathfrak{N}$.

Согласно определению $(20)$ оператора $W\left(L_{0}, L\right)$ и известным формулам, задающим действие $U(t)$ и $U_{0}(t)$ в представлении функциональной модели, для векторов $\mathscr{P}\left(\begin{array}{l}f \\ g\end{array}\right)$ из области определения $W\left(L_{0}, L\right)$ имеем

$$
\begin{array}{r}
J U(t) J^{-1} \mathscr{P}\left(\begin{array}{l}
f \\
g
\end{array}\right)=\mathscr{P} \exp (i k t)\left(\begin{array}{l}
f \\
g
\end{array}\right), \\
J U_{0}(t) J^{-1} W\left(L_{0}, L\right) \mathscr{P}\left(\begin{array}{c}
f \\
g
\end{array}\right)=\mathscr{P} \exp (i k t)\left(\begin{array}{l}
f \\
h
\end{array}\right) .
\end{array}
$$

При этом $(g-h) \in \mathrm{L}^{2}(\mathbb{R} ; E)$ и справедлива оценка (ср. с доказательством леммы 1$)$

$$
\begin{aligned}
& \left\|J U_{0}(t) U(-t) J^{-1} \mathscr{P}\left(\begin{array}{c}
f \\
g
\end{array}\right)-W\left(L_{0}, L\right) \mathscr{P}\left(\begin{array}{c}
f \\
g
\end{array}\right)\right\|_{\mathfrak{L}} \\
& =\left\|J U(-t) J^{-1} \mathscr{P}\left(\begin{array}{c}
f \\
g
\end{array}\right)-J U_{0}(-t) J^{-1} W\left(L_{0}, L\right) \mathscr{P}\left(\begin{array}{c}
f \\
g
\end{array}\right)\right\|_{\mathfrak{L}} \\
& =\left\|\mathscr{P} \exp (-i k t)\left(\begin{array}{c}
0 \\
g-h
\end{array}\right)\right\|_{\mathfrak{L}} \leqslant\left\|P_{+} \exp (-i k t)(g-h)\right\|_{L^{2}(\mathbb{R} ; E)} .
\end{aligned}
$$

Выражение, стоящее в правой части последнего неравенства, стремится к нулю, когда $t \rightarrow+\infty$. Таким образом, установлено, что на своей области определения оператор $W\left(L_{0}, L\right)$ представи́м в виде

$$
W\left(L_{0}, L\right) \mathscr{P}\left(\begin{array}{l}
f \\
g
\end{array}\right)=\lim _{t \rightarrow \infty} J U_{0}(t) U(-t) J^{-1} \mathscr{P}\left(\begin{array}{l}
f \\
g
\end{array}\right) .
$$


Далее, поскольку

$$
\sup _{\mathbb{R} \backslash\{0\}}\left\|S^{-1}(k)\right\|<\infty
$$

при выполнении условий предложения 4, то согласно [14] группа $U(t)$ равномерно ограничена на абсолютно непрерывном подпространстве оператора $L$. Как следствие этого оператор $J^{-1} W\left(L_{0}, L\right) J$ ограничен и продолжается по непрерывности на $J^{-1} \cos _{\mathfrak{H}} \mathfrak{N}=\mathscr{N}_{e}$. Полученный в результате этого продолжения ограниченньй оператор $\widetilde{\Omega}$ представи́м в виде

$$
\widetilde{\Omega} \varphi=\lim _{t \rightarrow \infty} U_{0}(t) U(-t) \varphi, \quad \varphi \in \mathscr{N}_{e}
$$

Согласно формулам (14) и (20) оператор $\widetilde{\Omega}$ является левым обратным к прямому волновому оператору $\Omega=\mathrm{s}-\lim _{t \rightarrow \infty} U(t) U_{0}(-t): \mathscr{H} \rightarrow \mathscr{N}_{e}$ и одновременно правьг обратным на подпространстве $\mathscr{N}_{e}$. Лемма доказана.

\section{§7. Факторизация скалярного кратного характеристической функции}

Согласно предложению 3 сушествует ограниченная аналитическая в $\mathbb{C}_{+}$оператор-функция $\Sigma(\lambda)$, удовлетворяющая соотношениям

$$
\Sigma(\lambda) S(\lambda)=S(\lambda) \Sigma(\lambda)=m(\lambda) I, \quad \operatorname{Im} \lambda>0,
$$

где $m(\lambda)=\operatorname{det}\left(I-(S(\lambda)-I)^{2}\right)$. Функция $m(\lambda) \in \mathrm{H}^{\infty}\left(\mathbb{C}_{+}\right)$допускает (см. [20]) внешне-внутреннюю каноническую факторизацию

$$
m(\lambda)=m_{1}(\lambda) \cdot m_{2}(\lambda) \cdot m_{3}(\lambda)
$$

Здесь $m_{1}(\lambda)$ - произведение Бляшке, построенное по нулям $m(\lambda)$ :

$$
m_{1}(\lambda)=\prod_{n} \frac{\lambda-\lambda_{n}}{\lambda-\bar{\lambda}_{n}}
$$

$m_{2}(\lambda)-$ сингулярная внутренняя функция вида

$$
m_{2}(\lambda)=e^{i a \lambda} \exp \left(i \int_{\mathbb{R}} \frac{t \lambda-1}{\lambda+t} d \mu(t)\right),
$$

где $\mu$ - конечная сингулярная мера и $a \geqslant 0 ; m_{3}(\lambda)$ - ограниченная в верхней полуплоскости внешняя функция, задаваемая следуюшей формулой

$$
m_{3}(\lambda)=e^{i \omega} \exp \left\{\frac{i}{\pi} \int_{\mathbb{R}} \ln (|m(\kappa+i 0)|) \frac{\kappa \lambda+1}{\lambda-\kappa} \frac{d \kappa}{1+\kappa^{2}}\right\}, \quad \omega \in \mathbb{R} .
$$

ЛЕмма 3. Пусть выполнень условия предложсения 3. Если $b(x) \notin \mathscr{E}$, то характеристическая функиия $S(\lambda)$ имеет скалярное кратное $m_{1}(\lambda) \cdot m_{3}(\lambda)$, әде $m_{1}(\lambda)$ - конечное произведение Бляшке, построенное по набору $\left\{\lambda_{n}\right\}=$ $\sigma_{p}(L) \subset i \mathbb{R}_{+}$. 
ДоКАЗАТЕльство. Нули определителя $m(\lambda)$ в верхней полуплоскости совпадают с собственными значениями оператора $L$. В самом деле, равенство $m(\lambda)=0$ означает, что $(S(\lambda)-I)^{2} \in \mathfrak{S}_{1}$ имеет собственное значение 1 . Поскольку $\|S(\lambda)\| \leqslant 1$, это равносильно тому, что $\operatorname{ker} S(\lambda) \neq\{0\}$. Согласно соотношению (13) последнее выполнено в том и только том случае, когда $-1 \in \sigma(Q(\lambda))$, что в силу (4) эквивалентно $\lambda \in \sigma_{p}(L)$. В силу предложения 2 при сделанных предположениях произведение Бляшке $m_{1}(\lambda)$ содержит конечное число дробно-линейных сомножителей.

Определитель $m(\lambda)$ продолжается по непрерывности на $\mathbb{R} \backslash\{0\}$, поскольку оператор-функция $Q(\lambda) \in \mathfrak{S}_{2}, \lambda \in \mathbb{C}_{+}$, допускает продолжение по непрерывности в классе $\mathfrak{S}_{2}$ на $\mathbb{R} \backslash\{0\}$ (см. доказательство предложения 3 ). Как отмечалось в доказательстве предложения 4 , при $\kappa \neq 0$ имеем $\operatorname{ker}(I+Q(\kappa+i 0))=\{0\}$ и, следовательно, $m(\kappa+i 0) \neq 0$, если $\kappa \in \mathbb{R} \backslash\{0\}$.

Согласно известному результату теории аналитических функций применительно к рассматриваемой ситуации внешний сомножитель $m_{3}(\lambda)$ одновременно с $m(\lambda)$ непрерывен вплоть до вещественной оси всюду, за исключением нуля, причем $\left|m_{3}(\kappa+i 0)\right|=|m(\kappa+i 0)|, \kappa \neq 0$. Отсюда следует, что $m_{2}(\lambda)$ продолжается по непрерывности на $\mathbb{R} \backslash\{0\}$, и поэтому носитель меры $\mu$ сосредоточен в нуле (см., например, [20; гл. 5]), т.е. сингулярный сомножитель в канонической факторизации $m(\lambda)$ имеет вид

$$
m_{2}(\lambda)=\exp \left(i a \lambda-i \frac{c}{\lambda}\right)
$$

где $a$ и $c$ - неотрицательные константы.

Покажем, что $m(i \tau) \rightarrow 1$ при $\tau \rightarrow \infty$. Для $\tau \geqslant 1$ справедливо неравенство $|q(x, y ; i \tau)| \leqslant|q(x, y ; i)|$, где $q(x, y ; i) \in \mathrm{L}^{2}(\mathbb{R} \times \mathbb{R})$, и, кроме того, $q(x, y ; i \tau) \rightarrow 0$, когда $\tau \rightarrow \infty$. По теореме Лебега о предельном переходе имеем

$$
\|Q(i \tau)\|_{\mathfrak{S}_{2}} \rightarrow 0, \quad \tau \rightarrow \infty
$$

Отсюда в силу (15) следует, что $\|S(i \tau)-I\|_{\mathfrak{S}_{2}} \rightarrow 0$ при $\tau \rightarrow \infty$ и, стало быть,

$$
m(i \tau)=\operatorname{det}\left(I-(S(i \tau)-I)^{2}\right) \rightarrow 1, \quad \tau \rightarrow \infty
$$

Последнее возможно лишш в том случае, если $a=0$. Иначе $m_{2}(i \tau) \rightarrow 0, \tau \rightarrow \infty$, что противоречит (22), поскольку в канонической факторизации $m(\lambda)$ сомножители $m_{1}(\lambda)$ и $m_{3}(\lambda)$ - ограниченные функции.

В силу условия $b(x) \notin \mathscr{E}$ оператор-функция $S^{-1}(\lambda)=(m(\lambda))^{-1} \Sigma(\lambda)$ равномерно ограничена в верхней полуокрестности точки $\lambda=0$ (см. доказательство предложения 4). Учитьвая ограниченность $m_{1}(\lambda)$ и $m_{3}(\lambda)$ в $\mathbb{C}_{+}$, заключаем, что оператор-функция $\left(m_{2}(\lambda)\right)^{-1} \Sigma(\lambda)$ аналитична и ограничена в верхней полуплоскости. Лемма доказана.

В следующем параграфе потребуются некоторые сведения об аналитических в круге сжимающих операторнозначных функциях (см. [16]). Ниже через $\mathrm{H}^{2}(\mathfrak{A})$ обозначается класс Харди аналитических в круге $\mathbb{D}=\{z \in \mathbb{C}:|z|<1\}$ функций $f(z)$ со значениями в гильбертовом пространстве $\mathfrak{A}$ таких, что

$$
\sup _{r<1} \int_{0}^{2 \pi}\left\|f\left(r e^{i \varphi}\right)\right\|_{\mathfrak{A}}^{2} d \varphi<\infty
$$


функции из $\mathrm{H}^{2}(\mathfrak{A})$ отождествляются с классами эквивалентности своих граничных значений на окружности $|z|=1$, образуюшими подпространство в $\mathrm{L}^{2}(\mathfrak{A})$.

Аналитическая в круге $\mathbb{D}$ сжимаюшая оператор-функция $\Theta(z)$, действующая из гильбертова пространства $\mathfrak{A}$ в гильбертово пространство $\mathfrak{A}^{\prime}$, называется внешней, если $\cos _{\mathrm{L}^{2}\left(\mathfrak{A}^{\prime}\right)} \Theta H^{2}(\mathfrak{A})=H^{2}\left(\mathfrak{A}^{\prime}\right)$. Оператор-функция $\Theta(z): \mathfrak{A} \rightarrow \mathfrak{A}^{\prime}$ называется внутренней, если отображение $\Theta$ из $\mathrm{H}^{2}(\mathfrak{A})$ в $\mathrm{H}^{2}\left(\mathfrak{A}^{\prime}\right)$ является изометрическим. Сжимающая оператор-функция $\Theta(z)$ называется *-внешней (*-внутренней), если аналитическая в $\mathbb{D}$ оператор-функция $\Theta^{*}(\bar{z}): \mathfrak{A}^{\prime} \rightarrow \mathfrak{A}$ является внешней (соответственно внутренней).

Всякая сжимающая аналитическая оператор-функция $\Theta(z): \mathfrak{A} \rightarrow \mathfrak{A}^{\prime}$ единственным образом представляется в виде $\Theta(z)=\Theta^{0}(z) \oplus$ const, где const означает изометрическую постоянную, а $\Theta^{0}(z)$ не содержит таких постоянных слагаемых. Оператор-функция $\Theta^{0}(z)$ назьвается чистой частью $\Theta(z)$.

\section{§8. Свойство полноты волновых операторов}

С помошью интеграла Рисса строится проектор

$$
P=-\frac{1}{2 \pi i} \int_{\Gamma} R(\lambda) d \lambda
$$

на линейную оболочку $\mathscr{H}_{d}:=P \mathscr{H}$ собственных векторов оператора $L$ параллельно подпространству $\mathscr{H}_{c}:=(I-P) \mathscr{H}$; здесь $\Gamma \subset \mathbb{C}_{+}-$положительно ориентированный замкнутый контур, разделяющий $\sigma_{c}(L)$ и $\sigma_{d}(L)$. Согласно [21] оператор $L$ разложи́м относительно прямой суммы $\mathscr{H}=\mathscr{H}_{c}+\mathscr{H}_{d}$ :

$$
P D(L) \subset D(L), \quad L \mathscr{H}_{c} \subset \mathscr{H}_{c}, \quad L \mathscr{H}_{d} \subset \mathscr{H}_{d}
$$

Ниже через $L_{c}$ обозначается сужение $L$ на подпространство $\mathscr{H}_{c}$.

ТЕОРема 2. Пусть выполнены условия теоремы 1 и, кроме того, $b(x)(\ln |x-y|)^{2} b(y) \in \mathrm{L}^{1}(\mathbb{R} \times \mathbb{R})$. Предположим, что оператор $L=L_{0}+i V$ вполне несамосопряженный $u \lim _{\tau \rightarrow 1} N(\tau b)=N(b) . \quad$ Тогда $\Omega \mathscr{H}=\mathscr{H}_{c}$, на подпространстве $\mathscr{H}_{c}$ существует обратный волновой оператор $\widetilde{\Omega}=\mathrm{s}-\lim _{t \rightarrow \infty} U_{0}(t) U(-t)$ и справедливо соотношение

$$
L_{c}=\Omega L_{0} \widetilde{\Omega}
$$

ДокАЗАтЕльство. 1) Сушествование прямого волнового оператора $\Omega=$ $\mathrm{s}-\lim _{t \rightarrow \infty} U(t) U_{0}(-t)$ установлено в теореме 1 . Построенный в лемме 2 левый обратный $\widetilde{\Omega}=\mathrm{s}-\lim _{t \rightarrow \infty} U_{0}(t) U(-t)$ является одновременно правьг обратным к $\Omega$ на абсолютно непрерьвном подпространстве $\mathcal{N}_{e}$ оператора $L$ и, таким образом, $\Omega \mathscr{H}=\mathscr{N}_{e}$. Ниже будет установлено, что подпространство $\mathscr{N}_{e}$ совпадает с $\mathscr{H}_{c}$.

Согласно [18] в рассматриваемой здесь ситуации $\mathscr{H}=\mathscr{N}_{e} \dot{+} \mathscr{N}_{i}$, причем проектор на $\mathscr{N}_{i}$ параллельно $\mathscr{N}_{e}$ ограничен (в силу предложения 4). Если $\varphi \in \mathscr{N}_{e}$, то вектор-функция $R(\lambda) \varphi$ аналитична в $\mathbb{C}_{+}\left(\right.$см. [14]) и, стало быть, $\varphi \in \mathscr{H}_{c}$, т.е. $\mathscr{N}_{e} \subset \mathscr{H}_{c}$. Покажем, что $\mathscr{N}_{i} \cap \mathscr{H}_{c}=\{0\}$, откуда будет следовать равенство $\mathscr{N}_{e}=\mathscr{H}_{c}$. 
2) Обозначим через $T=(L-i I)(L+i I)^{-1}$ преобразование Кэли диссипативного оператора $L$ и рассмотрим характеристическую функцию $\Theta_{T}(z)$ сжатия $T$ :

$$
\Theta_{T}(z)=-T+z\left(I-T T^{*}\right)^{1 / 2}\left(I-z T^{*}\right)^{-1}\left(I-T^{*} T\right)^{1 / 2},
$$

действуюшую из $\mathfrak{D}=\operatorname{clos} \mathscr{H}\left(I-T^{*} T\right)^{1 / 2} \mathscr{H}$ в $\mathfrak{D}^{\prime}=\operatorname{clos} \mathscr{H}\left(I-T T^{*}\right)^{1 / 2} \mathscr{H}$. Оператор-функция $\Theta_{T}(z): \mathfrak{D} \rightarrow \mathfrak{D}^{\prime}$ аналитична в круге $\mathbb{D}$, является там сжимающей и совпадает с $S(i(1+z) /(1-z))($ см. [16; гл. 9]). В соответствии с этим и вследствие леммы 3 оператор-функция $\Theta_{T}(z)$ обладает скалярным кратным $\alpha(z) \cdot \delta(z)$, где $\alpha(z)=m_{3}(i(1+z) /(1-z))$ - ограниченная в $\mathbb{D}$ внешняя функция, задаваемая формулой

$$
\alpha(z)=\exp \left\{\frac{1}{2 \pi} \int_{0}^{2 \pi} \ln \left(\left|m\left(-\operatorname{ctg} \frac{\varphi}{2}+i 0\right)\right|\right) \frac{e^{i \varphi}+z}{e^{i \varphi}-z} d \varphi\right\}
$$

а $\delta(z)=m_{1}(i(1+z) /(1-z))$ - конечное произведение Бляшке:

$$
\delta(z)=\prod_{n} \frac{z-z_{n}}{1-z_{n} z}, \quad z_{n}=\frac{\lambda_{n}-i}{\lambda_{n}+i} \in \mathbb{R} .
$$

Рассмотрим так называемую $*$-каноническую факторизацию характеристической функции $\Theta_{T}(z)$ (см. [16]):

$$
\Theta_{T}(z)=\Theta_{* e}(z) \Theta_{* i}(z),
$$

где $\Theta_{* e}(z)-*$-внешняя, $\Theta_{* i}(z)-*$-внутренняя оператор-функции. По теореме Сёкефальви-Надя и Фояша о внешне-внутренней факторизации оператор-функций, обладающих скалярньм кратным, множитель $\Theta_{* i}(z)$ имеет скалярное кратное $\delta(z)$. Вследствие теоремы о триангуляции сжатия, порожденной факторизацией его характеристической функции (см. [16; гл. 7]), подпространство $\mathscr{N}_{i}$ инвариантно относительно $T$ и характеристическая функция $\vartheta(z)$ сужения $T \mid \mathscr{N}_{i}$ совпадает с чистой частью $\Theta_{* i}(z)$, а стало быть, обладает скалярным кратным $\delta(z)$.

3) Подпространство $\mathscr{G}=\mathscr{N}_{i} \cap \mathscr{H}_{c}=(I-P) \mathscr{N}_{i}$ инвариантно относительно $T$ и $\mathscr{N}_{i}=\mathscr{H}_{d} \dot{+} \mathscr{G}$. Согласно общей теории функциональных моделей подпространству $\mathscr{G}$ отвечает факторизация характеристической функции $\vartheta(z)$ оператора $T \mid \mathscr{N}_{i}$ :

$$
\vartheta(z)=\vartheta_{2}(z) \vartheta_{1}(z)
$$

такая, что характеристическая функция $T \mid \mathscr{G}$ совпадает с чистой частью $\vartheta_{1}(z)$. При этом подпространство $\mathscr{G}$ имеет в $\mathscr{N}_{i}$ конечную коразмерность и чистая часть $\vartheta_{2}(z)$ представляет собой сжимающую оператор-функцию в конечномерном пространстве, обладающую скалярньм кратным. Поскольку в равенстве (23) левая часть $\vartheta(z)$ и сомножитель $\vartheta_{2}(z)$ обладают скалярными кратньми, то и $\vartheta_{1}(z)$ обладает скалярным кратным. А именно $\vartheta_{1}(z)$ обладает скалярным кратным $\delta(z)$ и, следовательно, является внутренней оператор-функцией (см. [16; гл. 5]).

Учитьвая, что $\sigma\left(L_{c}\right) \subset \mathbb{R}$, имеем $\sigma\left(T \mid \mathscr{H}_{c}\right) \cap \mathbb{D}=\varnothing$ и, стало быть, $\sigma(T \mid \mathscr{G}) \cap$ $\mathbb{D}=\varnothing$; поэтому характеристическая функция $T \mid \mathscr{G}$, совпадающая с чистой частью $\vartheta_{1}(z)$, обратима в $\mathbb{D}$. Таким образом, оператор-функция $\vartheta_{1}(z)$ обратима всюду в $\mathbb{D}$ и обладает скалярным кратным $\delta(z)$. Отсюда следует, что $\vartheta_{1}^{-1}(z)$ равномерно 
ограничена и аналитична в $\mathbb{D}$ и, стало быть, $\vartheta_{1}(z)$ - внешняя. Оператор-функция, внутренняя и внешняя одновременно, является унитарной константой, что применительно к $\vartheta_{1}(z)$ возможно лишш в случае, когда $\mathscr{G}=\{0\}$.

4) Далее, согласно теореме 1 оператор $\Omega$ сплетает $L$ и $L_{0}$ :

$$
L \Omega=\Omega L_{0}, \quad \Omega D\left(L_{0}\right) \subset D\left(L_{0}\right)=D(L) .
$$

Поскольку $\Omega \mathscr{H}=\mathscr{H}_{c}$ и $\Omega D\left(L_{0}\right) \subset D\left(L_{0}\right)$, то $\Omega D\left(L_{0}\right) \subset D\left(L_{0}\right) \cap \mathscr{H}_{c}$. Покажем, что $\Omega D\left(L_{0}\right)=D\left(L_{0}\right) \cap \mathscr{H}_{c}$.

Отметим, что $R(\lambda) \mathscr{H}_{c} \subset \mathscr{H}_{c}$ и, следовательно, $U(t) \mathscr{H}_{c} \subset \mathscr{H}_{c}$. Ввиду этого для произвольных $t>0$ и $\psi \in \mathscr{H}_{c}$ справедливо соотношение

$$
\widetilde{\Omega} \psi=U_{0}(-t) \widetilde{\Omega} U(t) \psi
$$

и, таким образом, $U_{0}(t) \widetilde{\Omega}=\widetilde{\Omega} U(t)$. Выполним предельный переход при $t \downarrow 0$ в равенстве

$$
\widetilde{\Omega} \frac{U(t)-I}{t} \psi=\frac{U_{0}(t)-I}{t} \widetilde{\Omega} \psi,
$$

где $\psi \in D\left(L_{0}\right) \cap \mathscr{H}_{c}$. Поскольку $L\left(D(L) \cap \mathscr{H}_{c}\right) \subset \mathscr{H}_{c}$, то предел слева сушествует и равен $i \widetilde{\Omega} L \psi$. Одновременно сушествует предел справа

$$
\lim _{t \downarrow 0} \frac{U_{0}(t)-I}{t} \widetilde{\Omega} \psi=i L_{0} \widetilde{\Omega} \psi
$$

причем $\widetilde{\Omega} \psi \in D\left(L_{0}\right)$. Таким образом, $\widetilde{\Omega}\left(D\left(L_{0}\right) \cap \mathscr{H}_{c}\right) \subset D\left(L_{0}\right)$ и, стало быть, $D\left(L_{0}\right) \cap \mathscr{H}_{c} \subset \Omega D\left(L_{0}\right)$.

В результате установлено совпадение $\Omega D\left(L_{0}\right)=D\left(L_{0}\right) \cap \mathscr{H}_{c}$. С учетом этого из сплетаюшего соотношения $L \Omega=\Omega L_{0}$ следует, что $L \psi=\Omega L_{0} \widetilde{\Omega} \psi$ для произвольного $\psi \in D\left(L_{0}\right) \cap \mathscr{H}_{c}$, т.е. $L_{c}=\Omega L_{0} \widetilde{\Omega}$. Теорема доказана.

\section{Список литературы}

1. Карлеман Т. Математические задачи кинетической теории газов. М.: ИЛ, 1960.

2. Боголюбов H. Н. Проблемы динамической теории в статистической физике. М.: Гостехиздат, 1946.

3. Владимиров В. С. Математические задачи односкоростной теории переноса нейтронов // Труды МИАН. 1961. Т. 61. С. 1-158.

4. Черчиньяни K. Теория и приложения уравнения Болњцана. М.: Мир, 1978.

5. Кейз К., Цвайфель П. Линейная теория переноса. М.: Мир, 1972.

6. Шихов С. Б. Вопросы математической теории реакторов. Линейньй анализ. М.: Атомиздат, 1973.

7. Рихтмайер P. Принципы современной математической физики. М.: Мир, 1982.

8. Lehner J. The spectrum of the neutron transport operator for the infinite slab // J. Math. Mech. 1962. V. 11. № 2. P. 173-181.

9. Lehner J., Wing G.M. On the spectrum of an unsymmetric operator arising in the transport theory of neutrons // Comm. Pure Appl. Math. 1955. V. 8. P. 217-234.

10. Lehner J., Wing G.M. Solution of the linearized Boltzmann transport equation for the slab geometry // Duke Math. J. 1956. V. 23. P. 125-142.

11. Куперин Ю. А., Набоко С. Н., Романов Р. В. Спектральньй анализ односкоростного оператора переноса и функциональная модель // Функц. анализ и его прилож. 1999. T. 33. №3. C. $47-58$. 
12. Степин $C$. A. Возмущение спектра и задача рассеяния в односкоростной теории переноса // Деп. в ВИНИТИ. 07.02.2000. № 285-В00. Москва, 2000.

13. Kato T. Wave operators and similarity for some non-selfadjoint operators // Math. Ann. 1966. V. 162. P. 258-279.

14. Набоко $C . H$. Функциональная модель теории возмущений и ее приложения к теории рассеяния // Труды МИАН. 1980. Т. 147. С. 86-114.

15. Степин $C$. A. Возмущение спектра и волновые операторы в линейной теории переноса // УМH. 1999. Т. 54. № 5. С. 175-176.

16. Сёкефальви-Надь Б., Фояи Ч. Гармонический анализ операторов в гильбертовом пространстве. М.: Мир, 1970.

17. Рид М., Саймон Б. Методы современной математической физики. Т. 3. М.: Мир, 1982.

18. Павлов Б. С. Об условиях отделимости спектральных компонент диссипативного оператора // Изв. АН СССР. Сер. матем. 1975. Т. 39. №1. С. 123-148.

19. Павлов Б. С. Самосопряженная дилатация диссипативного оператора Шрёдингера и разложение по его собственньм функциям // Матем. сб. 1977. Т. 120. № 4. С. 511-536.

20. Гофман К. Банаховы пространства аналитических функций. М.: ИЛ, 1963.

21. Като Т. Теория возмущений линейных операторов. М.: Мир, 1972.

Московский государственньй

Поступила в редакцию

университет им. М.В. Ломоносова

26.04 .2000 